\title{
Adjunctive antibiotics in the treatment of generalized aggressive periodontitis
}

\author{
Can an adjunctive course of metronidazole and amoxicillin improve out- \\ comes in people who have generalised aggressive periodontitis?
}

\begin{abstract}
Guerrero A, Griffiths GS, Nibali L, Suvan J, Moles DR, Laurell
L, Tonetti MS. Adjunctive benefits of systemic amoxicillin and metronidazole in non-surgical treatment of generalized aggressive periodontitis: a randomized placebo-controlled clinical trial. J Clin Periodontol 2005; 32:1096-1107.
\end{abstract}

Design The study was a randomised controlled trial.

Intervention People who had generalised aggressive periodontitis (GAP) underwent a standard cycle of periodontal therapy (oral hygiene instruction, supra- and subgingival mechanical instrumentation of root surface) following which members of the test group took an adjunctive course of systemic antibiotics (500 mg metronidazole and $500 \mathrm{mg}$ amoxicillin) for 7 days and the control group received a placebo. Reassessment visits occurred 2 and 6 months post-treatment.

Outcome measure The primary outcome measure was probing pocket depth (PPD) reduction in sites with an initial PPD $>7 \mathrm{~mm}$. The secondary outcome measure was changes in other clinical parameters.

Results A total of 41 subjects took part in the study, with one lost to follow-up between the 2- and 6-month reassessments. In both groups all clinical parameters improved at 2 and 6 months. The test group showed greater reduction in PPD than the control group in terms of fullmouth PPD and life accumulative attachment loss. Seventy-four percent of pockets with PPD $>5 \mathrm{~mm}$ at baseline were $<4 \mathrm{~mm}$ at 6 months in the test group compared with $54 \%$ in the control group $(P=0.008)$.

Conclusions A 7-day adjunctive course of metronidazole and amoxicillin improved short-term clinical outcomes in people who had GAP.

\section{Commentary}

Although GAP affects a small proportion of the general of the population, it is a disease of high morbidity. Formerly known as generalised juvenile periodontitis, GAP generally affects younger adults who otherwise appear healthy, and the most distinguishing feature is the rapid rate of attachment loss. This destruction tends to occur episodically, affecting several areas of the mouth, and tends also to have a familial aggregation. There is commonly a higher rate of cases refractory to treatment than in those of chronic periodontitis, probably because of the high prevalence of Actinobacillus actinomycetemcomitans and other virulent pathogens.

Guerrero and colleagues' RCT examines the effect of full-mouth periodontal debridement completed within $24 \mathrm{~h}$, followed by a $0.2 \%$ chlorhexidine rinse twice daily for 2 weeks post-treatment. (This approach was first documented by Quirnyen et al., ${ }^{1}$ but does not appear to be repeated by other groups. Nonetheless, it seems to be a reasonable method.) This was followed by $500 \mathrm{mg}$ each of the antibiotics metronidazole and amoxicillin, three times daily for 7 days.

This is a well-designed study, with blinded randomisation, placebo controls and intent-to-treat analysis. The groups were stratified for two significant confounding variables, smoking and baseline PPD. What is most unique about this study, however, is its analysis of the data. Most periodontal clinical trials report mean changes in full mouth PPD and/or attachment levels. As the authors correctly point out, this can underestimate real clinical changes in areas of more advanced disease. The authors examined outcomes of more clinical importance than are traditionally reported, ie, mean level of clinical attachment gain and PPD reduction in initially deep pockets and the proportion of sites with $2 \mathrm{~mm}$ or more attachment gain. Thus, when compared with the full mouth nonsurgical therapy alone, the antibiotic regimen resulted in a difference of both clinical and statistical significance.

Despite the rigour of the study design and the elegance of the statistical analysis, as a clinician, one must consider the results of this study in light of the risks of antibiotic therapy. Hafajee ${ }^{2}$ recently suggested that several questions should be posed prior to prescribing antimicrobials as an adjunctive to periodontal therapy. Although the bulk of evidence suggests that adjunctive antibiotics are a valuable tool in managing aggressive forms of periodontal disease, the optimum dosages, duration and timing of antibiotic administration in relation to mechanical debridement remain unclear. Guerrero et al. here used higher doses of both metronidazole and amoxicillin than employed previously.

Not surprisingly, the incidence of adverse reactions was much higher than in the placebo group (65\% versus $23 \%)$. One must also consider the potential for development of antibiotic-resistant microorganisms. How do these outcomes compare with a $14 \%$ reduction in the need for periodontal surgical intervention? As with all clinical decisions, each individual patient's medical and dental history, together with the clinician's expertise and judgement, must be balanced with the sound evidence presented in this clinical trial.

\section{Practice point In people diagnosed with GAP:}

there may be some clinical benefit over nonsurgical therapy alone of $500 \mathrm{mg}$ metronidazole plus $500 \mathrm{mg}$ amoxicillin three times daily for 7 days, in addition to nonsurgical periodontal therapy and a $0.2 \%$ chlorhexidine rinse twice daily for 2 weeks postdebridement; and

the risks of antibiotic therapy as an addition to traditional nonsurgical therapy must be carefully weighed against any potential benefits.

\section{Debora C Matthews}

Division of Periodontics, Dalhousie University, Halifax, Nova Scotia, Canada

1. Quirnyen M, Bollen CM, Vandekerckhove BN, Papaioannou W, Eyssen H. Full- vs. partial-mouth disinfection in the treatment of periodontal infections; short-term clinical and microbiological observations. J Dent Res 1995; 74:1459-1467.

2. Haffajee AD. Systemic antibiotics: to use or not to use in the treatment of periodontal infections. That is the question. J Clin Periodontol 2006; 33:359-361.

Evidence-Based Dentistry (2006) 7, 67.

doi:10.1038/sj.ebd.6400424 